\title{
Rouge violent et noir mortel dans les romans pour adolescents de Guillaume Guéraud : une écriture sans contrainte
}

\author{
Valérie Alfvén \\ Université de Stockholm
}

Les propos de Livres Hebdo en 2006 à propos de Guillaume Guéraud et de son livre Je mourrai pas gibier, alors fraîchement paru aux éditions du Rouergue, donnent le ton: "Animées d'une rage insolente, sans graisse sentimentale, carburant à l'émotion forte, les histoires de cet écrivain de 34 ans, dit "pour la jeunesse", [...] brillent d'une noire fureur. » On le voit, Guéraud fait partie de ces auteurs qui posent problème en littérature jeunesse. La crudité du langage, la noirceur de la 
société dépeinte, la violence décrite sont pour certains excessives. En quelque sorte, les livres de Guillaume Guéraud dépassent les contraintes habituelles de la littérature jeunesse par la violence affichée et défient l'idylle ${ }^{1}$ en écrivant des fins parfois malheureuses ou en tout cas sans espoir. Ses romans mêlés de rouge (violence) et de noir (mort / absurde) brisent un « horizon d'attente ${ }^{2}$ » et se confrontent à une doxa littéraire qui s'appuie sur la loi française de juillet 1949. Cette loi relative aux publications pour la jeunesse insiste sur l'importance de la «non-démoralisation » du jeune ${ }^{3}$. Mais peut-on déclarer que les romans de Guéraud «démoralisent» le lecteur adolescent en l'incitant à la débauche? Quelle place est faite au rouge -

\footnotetext{
${ }^{1}$ Idylle : nous faisons référence à la définition de Bakhtine, qui renvoie aux stéréotypes heureux et idéalisant de l'idylle dans les relations amoureuses ou à travers l'idylle de la patrie par exemple. Sans idylle, le lecteur assiste à une "déshumanisation » et à une «négation de tous les principes moraux [...] une désagrégation [...] de toutes les relations humaines d'autrefois: amour, famille, amitié, etc. L'homme positif du monde idyllique devient comique, pitoyable et superflu » $(1975$, p. 376$)$.

${ }^{2}$ L'horizon d'attente, tel que Jauss (1978) le conçoit constitue chez le lecteur, le récepteur du texte, un ensemble d'attentes et de règles qu'il a construit par ses lectures antérieures. Cependant, nous ajoutons que, dans le contexte de la littérature jeunesse, cet horizon d'attente se double souvent d'une fonction morale. La littérature jeunesse a une fonction didactique qui influence l'horizon d'attente du lecteur et ce dernier s'attend à retrouver dans le texte ces valeurs morales et didactiques.

${ }^{3}$ Article 2 de la loi du 16 juillet 1949 sur les publications destinées à la jeunesse, modifié par la loi du 9 juillet 2010 : «Les publications visées à l'article $1^{\text {er }}$ ne doivent comporter aucune illustration, aucun récit, aucune chronique, aucune rubrique, aucune insertion présentant sous un jour favorable le banditisme, le mensonge, le vol, la paresse, la lâcheté, la haine, la débauche ou tous actes qualifiés de crimes ou délits ou de nature à démoraliser l'enfance ou la jeunesse, ou à inspirer ou entretenir des préjugés ethniques ou sexistes. Elles ne doivent comporter aucune publicité ou annonce pour des publications de nature à démoraliser l'enfance ou la jeunesse. »
} 
violence sanguinaire - et au noir - mort des héros - dans ces récits? Quelles sont les motivations de l'auteur? Quels problèmes ces romans peuvent-ils poser étant donné le public adolescent auquel ils sont destinés et le chemin parcouru à travers différents prescripteurs (éditeurs, parents, bibliothécaires, enseignants, libraires, critiques) avant de l'atteindre?

Pour tenter de répondre à ces questions, nous nous pencherons sur trois romans guéraudiens parus successivement aux éditions du Rouergue en 2006, 2007 et 2009. Y être édité au début des années 2000 n'est pas anodin. En effet, cette maison d'édition a délibérément choisi de ne pas apposer la mention référant à la loi de juillet 1949 sur la protection des publications pour la jeunesse. Ce faisant, elle conserve une plus grande liberté d'action et peut alors se permettre de publier des textes réduisant les contraintes d'écriture liées à des sujets dits sensibles, voire tabous. Par ce biais, l'éditeur est également en mesure de protéger son auteur d'éventuelles poursuites judiciaires et de réduire le risque d'autocensure.

\section{Trois romans rouges et noirs}

Je mourrai pas gibier paraît en 2006. Ce roman a été distingué dans la catégorie "Romans adolescents » par le Prix Sorcières 2007. Il a même donné suite à une adaptation scénique (Boulogne-sur-mer, 2009) puis à une version en bande dessinée (Alfred, éditions Delcourt, coll. " Mirages », 2009) qui reçut en 2011 le «prix littéraire des lycéens et apprentis de la région Provence-Alpes-Côte d'Azur ». Classé comme un roman à lire à partir de 13-14 ans, il raconte l'histoire de Martial, un adolescent qui vient d'un petit village nommé Mortagne. Ce 
village est scindé en deux clans rivaux qui se font la guerre, ceux qui travaillent dans les vignes et ceux qui travaillent à la scierie. Cette scission met en lumière un village où se mêlent alcoolisme, brutalités et patriarcat. Martial a préféré s'éloigner de cette violence sociale sous-jacente en s'engageant dans la voie de la mécanique et en choisissant de vivre au pensionnat en semaine et à Mortagne le week-end. Le week-end, il fréquente de temps en temps le simplet du village, surnommé le « pleu-pleu », sur lequel la violence de son frère aîné Arnaud et de Frédo, l'ami de ce dernier, se déchaîne. Le personnage du «pleu-pleu » devient ainsi le prototype de la victime émissaire qu'évoque René Girard (1972) : par sa faiblesse et sa différence, il est extérieur au groupe, ce qui lui confère une fonction «sacrifiable ». Martial retrouve un matin le «pleu-pleu » sur le sol de sa maison littéralement massacré. Sans doute révolté ou abasourdi par la violence qui l'entoure et la brutalité des siens (le lecteur ne peut qu'émettre des suppositions), il se résout à emprunter la carabine de son père, le jour du mariage de son frère, pour violemment massacrer sa famille. Il finit par se défenestrer, sans pour autant réussir son suicide.

La Brigade de l'œil paraît en 2007. Avec ce polar futuriste, Guéraud ne désarme pas. En 2037, Rush Island est sous la dictature de l'impératrice Harmony et de la loi Bradbury qui interdit depuis vingt ans toute image sur le territoire. La brigade de l'œil, une unité dépendant du ministère de la Sécurité chargée de la répression, détruit toutes les images et brûle les yeux des délinquants, sans procès. À Rush Island, 15 \% de la population est aveugle. Kao, le jeune héros de 15 ans, fait un trafic d'images illégales et risque gros à chaque fois. Il entre dans la résistance et trouve un jour une réserve oubliée de vieux films, les derniers qui existent. Kao et son équipe de 
résistants sont alors traqués par la brigade de l'œil qui les retrouve et les extermine. L'allusion au roman de Ray Bradbury Farenheit 451 n'a pas échappé au lecteur et l'écriture de Guéraud se fait ici clairement cinématographique.

Avec Déroute sauvage, livre sanglant paru en 2009, nous progressons dans le degré de violence. Cette déroute est l'histoire de jeunes lycéens partis en voyage scolaire. Sur la route du retour, leur bus a un accident. Quelques élèves survivent mais ils vont être atrocement massacrés au fur et à mesure par trois êtres ultraviolents, à la limite du surnaturel et assoiffés de sang. Au lecteur d'imaginer à la fin si les deux élèves restants vont survivre à l'inhumanité dont ils ont été témoins.

Ce résumé des trois récits nous montre que la violence constitue un motif guéraudien fondateur, qui s'étend d'ailleurs à l'ensemble de son œuvre. Sur la trentaine de romans édités à ce jour, seuls deux livres connaissent une fin heureuse : La Fille la plus belle de la planète et une œuvre de commande d'obédience largement autobiographique, Sans la télé.

\section{Motivations de l'auteur}

Pour quelle/s raison/s la violence joue-t-elle un rôle central chez Guéraud? Pourquoi devient-elle moteur de l'action? S'interroger sur les motivations de l'auteur, c'est partir à la recherche d'une éventuelle autocensure que l'on rencontre très régulièrement chez les auteurs pour la jeunesse mais aussi se confronter à la question de la responsabilité d'un auteur face à son jeune lecteur ${ }^{4}$. Comme nous l'avons déjà souligné, la France

\footnotetext{
${ }^{4}$ Voir à ce sujet Collectif (2009).
} 
est un pays doté d'un appareil juridique (loi de juillet 1949 sur les publications destinées à la jeunesse ${ }^{5}$ ) qui potentiellement lui permet de condamner un auteur jeunesse pour "démoralisation ». Le rôle joué par la Commission de surveillance et par cette loi rend possible un certain contrôle sur la littérature jeunesse. Il est alors souvent difficile pour un auteur jeunesse de ne pas s'autocensurer dès l'écriture même du texte afin de se garantir une place au sein du champ de la littérature jeunesse sans créer de vagues. Guéraud, lui, se fait d'emblée reconnaître comme un auteur jeunesse de qualité mais aussi très dérangeant, à l'écriture souvent qualifiée de «coup de poing » ou encore qui "ne retient pas ses coups». Ses romans «ne laissent pas indifférents » : le sang y gicle de toute part et la mort, qui est toujours violente et souvent gratuite, fait partie intégrante de la vie de ses héros. Beaucoup critiqué, et de façon parfois hostile, par les prescripteurs émus par la violence de l'histoire et du ton, Guéraud est pourtant un auteur qui a trouvé son public et semble bien rejoindre les attentes de ce lectorat adolescent, pour qui l'image fait partie du quotidien. Notre auteur ne cache d'ailleurs pas son amour du septième art, pas plus qu'il ne tait l'influence évidente du cinéma sur son écriture. Il écrit, ou plutôt décrit, les scènes comme si lui-même avait l'œil derrière la caméra, par petits tableaux en phrases très courtes, phénomène relevé par un critique de Télérama à la parution de La Brigade de l'œil : " ce roman très sombre [...] est un hymne au cinéma aussi bien qu'à la littérature » (Abescat, 2007). Pour Je mourrai pas gibier, Guéraud explique qu'il s'est inspiré du film (ultraviolent) de Sam Peckinpah, La Horde sauvage (The Wild Bunch, 1969).

${ }^{5}$ Pour plus d'information sur cette loi, voir Crépin et Crétois (2003). 
L'auteur se laisse guider par ses propres goûts et envies sans se contraindre. Dans un entretien, il exprime clairement qu'il ne souhaite pas se censurer : «Quand j'écris, je ne me pose pas la question du lecteur et de son âge. Je mets en scène des personnages, et ce que je dois faire, c'est les mener au bout d'une histoire. » (Éditions du Rouergue, p. 8) Avec Je mourrai pas gibier, il avait tout simplement envie, gratuitement, de raconter une fusillade : «parce que c'est beau, une fusillade ${ }^{6}$ ». Les motivations de l'auteur sont donc purement personnelles et Guéraud ne s'attache pas une éventuelle responsabilité vis-à-vis de son public. Sa volonté est que ses livres et ses personnages «collent aux ados» et il conclut que «les scènes de violence donnent beaucoup de force à un livre» (ibid.). À partir du moment où la volonté de se dégager de toute contrainte autocensurante est affirmée, il peut laisser libre cours à l'écriture du rouge dans ses romans.

\section{La violence sous le signe du rouge}

Daniel Delbrassine constate que «[la] représentation de la violence dans le roman adressé aux adolescents a manifesté récemment une audace qui n'a plus rien à envier aux textes les plus durs de la littérature générale » (2006, p. 315). Guéraud ose donc et va plus loin dans l'audace que beaucoup de ses contemporains. Motifs omniprésents de ses textes, le rouge et la violence qu'il symbolise structurent la narration et l'écriture : phrases courtes, oralité. Dans ces trois romans, la violence est

\footnotetext{
${ }^{6}$ Réponse à une question posée à l'auteur à Paris en janvier 2012 lors d'un stage sur la «violence dans les livres de jeunesse » organisé par l'association de formation des bibliothécaires Lecture jeunesse.
} 
souvent " gratuite » : elle revêt un caractère imprévisible et ne semble pas a priori motivée par une raison particulière. Nous avons vu que, dans Je mourrai pas gibier, Martial se déchaîne à la suite de la découverte du corps massacré du pleu-pleu, mais s'il s'agit bien d'un élément déclencheur, le lecteur comprend que ce n'est pas uniquement cette découverte qui l'a poussé à la violence. Ce n'était pas la première fois qu'il découvrait le simplet victime de la violence de son frère. Martial massacre donc sa famille sans que le lecteur ne puisse expliquer exactement pourquoi. Avec le recul, le lecteur peut supposer que c'est un ensemble d'éléments combinés qui ont poussé Martial à l'acte: le protagoniste-narrateur évolue dans un contexte social et un milieu familial ultraviolents. La chasse est un modus vivendi et elle contribue à donner un aspect menaçant aux bagarres entre villageois. Le frère de Martial va régulièrement, avec l'un de ses amis, tabasser le "pleu-pleu » du village par pure méchanceté ou simple défoulement après une soirée arrosée ou une frustration. Pour autant, comme le montre cet extrait du premier chapitre, l'éventuelle culpabilité des personnages ne fait pas partie du tableau, même pour Martial qui vient d'abattre la plupart des membres de sa famille :

Je ne peux plus faire de mal à personne, maintenant. Même pas à moi. Ils m'ont ôté mes lacets et ma ceinture.

Ils ne tiennent pas à ce que je me foute en l'air. Mais, n'importe comment, il y a toujours un moyen. Le plus pratique aurait été avec le fusil que j'ai utilisé pour dégommer tout le monde. J'avais d'ailleurs prévu de conserver deux cartouches pour ma pomme. Sauf que, j'ai dû me laisser emporter par l'euphorie, je les ai toutes tirées. (Guéraud, 2006, p. 6)

La violence, dans ces trois romans, est principalement physique et s'accorde bien à la langue crue de Guéraud. Sa 
présence sémantique se déploie en permanence tout au long de la trame narrative et de manière ostentatoire. Prenons par exemple la présence du sang, explicite ou suggérée métaphoriquement comme dans cet extrait :

La tête de Frédo a fini par basculer sur la droite, libérant la pelle coincée dans les chairs de son cou, ouvrant une plaie béante par laquelle des geysers de sang se sont mis à fuser de sa carotide. Il s'est écroulé par terre alors que son sang continuait d'asperger le mur et ma sœur a commencé à hurler. Un marteau traînait sur l'établi.

Les cris de ma sœur me vrillaient les tympans alors je me suis emparé du marteau et je l'ai abattu sur sa bouche jusqu'à ce qu'elle se taise. (Guéraud, 2006, p. 55-56)

La suggestion de la violence passe par la présence des armes (dans les trois romans), qui inscrivent la violence dans le réel et renvoient parallèlement aux meilleurs films d'horreur : fusil, gigantesque maillet, serpes, poignards, pelle, marteau, machettes (plus ou moins rouillées), couteaux, chalumeaux, jusqu'aux armes de fantasy comme les galiscopes (lunettes qui forcent les brigadiers de l'œil à garder les yeux ouverts), ou les pyroculis (appareils pour brûler les yeux), ne sont que quelques exemples d'une longue liste. Comme nous allons le voir, ces armes participent d'une thématique et d'une esthétique du démantèlement.

\section{Thématique et esthétique du démantèlement}

Démantèlement des corps d'abord. Dans Déroute sauvage, le plus extrême des trois romans, il n'est plus possible de compter les plaies béantes, les ventres explosés ou empalés, les troncs dépecés, les poumons éclatés ou les estomacs perforés. Guéraud use du rouge jusqu'au grotesque; il jouit d'un macabre excessif 
dans la représentation rouge, faisant alors déraper la mort vers l'humour noir. La répulsion créée permet alors, étonnamment peut-être, une dédramatisation de la violence sordide. La plupart des passagers ont été " écrasés-découpés-concassés » (p. 39) et le tout baigne, selon la logique du genre, dans des mares de sang, comme dans ce court extrait:

Seul Marc vit nettement le crâne de Claire éclater. Mais même lui ne comprit pas. [...] Malgré les fragments de cervelle et les esquilles qui se dispersèrent. Malgré le sang qui éclaboussa son propre visage [...] son corps sans tête [...]. (Guéraud, 2009, p. 62)

Rien n'est épargné au lecteur, pas même une difficile scène d'actes de torture sur une jeune adolescente. La violence est partout et, comme au cinéma, elle comporte même du son : le lecteur peut entendre la pelle de Martial s'arrêter dans « un grand craquement en butant contre [1] es vertèbres cervicales " (Guéraud, 2006, p. 56). Les descriptions tendent à l'extrême, le lecteur comprenant rapidement que Guéraud utilise les codes du genre gore. Le sommet est atteint dans Déroute sauvage, où le gore se mêle au sadisme. Ce qui ajoute au malaise, c'est que les jeunes lycéens tout comme les adultes subissent le même sort. Pour le lecteur, la représentation de la violence se situe ainsi à la limite de l'abjection, au sens où l'entend Julia Kristeva : "Ça sollicite, inquiète, fascine le désir qui pourtant ne se laisse pas séduire.» (1980, p.9). L'abjection instaure un malaise, voire une nausée du lecteur face à ce déchaînement de violence auquel il assiste mais qui, finalement, l'attire aussi. La violence que propose Guéraud est étonnamment physique, brute et sans profondeur psychologique, ce qui peut la rendre d'autant plus effrayante. Elle semble avoir perdu son sens et s'être déchargée de toute notion de culpabilité. En effet, si Martial pleure après sa tuerie, ce n'est pas par sentiment de 
culpabilité, voire de libération ou de jouissance, mais simplement parce qu'il s'est fait mal au genou. Dans un contexte de littérature jeunesse, cette violence sans sens moral clairement explicité aux jeunes lecteurs provoque une résistance du côté des prescripteurs. Si la violence brute des romans guéraudiens témoigne clairement d'une ironie noire, nous pouvons également considérer qu'elle opère ici sur le mode de l'exorcisme, dès lors que la connaissance de la violence, «parce qu'elle permet un recul de l'affect, autorise alors le triomphe du spectateur sur la violence» (Marzano, p. 552).

Le démantèlement, chez Guéraud, n'est pas seulement physique, il est également d'ordre narratif, comme l'illustre l'emploi de phrases courtes et incisives, qui renvoient à une écriture cinématographique, par plans. La violence semble si imbriquée et indissociable de la narration qu'elle en devient abjecte parce qu'elle est, comme le rappelle Kristeva, « ce qui perturbe une identité, un système, un ordre. Ce qui ne respecte pas les limites, les places, les règles » (1980, p. 12) Le lecteur ressent donc un sentiment d'abjection lorsque son système de référence, individuel et social, est bouleversé. Publiant en littérature jeunesse, Guéraud franchit largement les limites de cet ordre préétabli ainsi que celles de l'horizon d'attente des prescripteurs et/ou du lecteur adolescent. La présence du sang ne provoque pas à elle seule l'abjection, elle opère une mise en abyme de la violence dans la narration dans laquelle la violence est imbriquée. La structure des phrases se fait écho du contenu et intensifie l'angoisse du lecteur à chaque point final comme à la fin du chapitre 10, où Martial (qui a déjà assassiné sa sœur et Frédo, l'ami de son frère) s'installe à son poste de tir pour 
continuer la tuerie depuis la chambre de ses parents, au premier étage :

J'ai voulu vérifier quelque chose. Je me suis collé le canon du fusil dans le visage en glissant un doigt dans la gâchette pour voir si je pouvais me tirer dessus tout seul. Je pouvais.

Alors j'ai repris l'arme normalement.

J'ai enclenché deux cartouches.

J'ai refermé le fût.

Et j'ai ouvert la fenêtre. (Guéraud, 2006, p. 62)

\section{Le noir comme finalité du rouge}

Qui dit «violence » chez Guéraud sous-entend «mort». Dans ses romans, les personnages meurent, sans différenciation, les adultes comme les adolescents. Les survivants ont comme dernière issue ou le suicide ou la folie. Dans Je mourrai pas gibier, le narrateur, Martial, après avoir sordidement massacré une grande partie de sa famille, se défenestre; Kao, le héros de La Brigade de l'œil, finit brûlé par la brigade de répression; la vie de Romain, sérieusement blessé dans Déroute sauvage, ne tient plus qu'à un fil et il semble glisser dans la folie après son horrible aventure, en laissant le lecteur sur cette dernière image de l'adolescent bien mal en point partant seul sur le chemin, image qui rappelle le final des 400 coups de Truffaut, ou celui du Bon, la brute et le truand de Sergio Leone. Guéraud, bien qu'il soit souvent décrié, ne déroge pourtant pas à l'ensemble des autres romans pour adolescents contemporains. En effet, comme le soulignent Danielle Thaler et Alain Jean-Bart, « la mort n'est plus l'événement qui initie l'aventure, mais elle appartient à cette aventure » (2002, p. 192). Dans les romans contemporains pour adolescents, il faut vivre avec la mort, qui est devenue banale. 
Guéraud prend alors le contre-pied d'une écriture de l'idylle et, par la mort de ses personnages, remet en question le principe de fin heureuse. Dans la longue tradition de la littérature jeunesse, la fin qui se termine en happy end repose sur une fonction bien précise. D'ordre moral, l'idylle tente de faire oublier la méchanceté (qui prend ici la forme de violence gratuite) commise par des personnages de l'histoire et de restaurer un ordre. Elle a une fonction réparatrice et salvatrice en contenant et en limitant la méchanceté de certains personnages. Elle permet un contrôle qui va dans le sens de la morale et d'un horizon d'attente. Comme le relève Bertrand Ferrier (p.50-51), elle fournit également une immunité à l'auteur, qui se met à l'abri de la loi de 1949 évoquée plus haut en ne «démoralisant» pas le lecteur, cette perte de l'espoir créant des résistances fortes chez certains critiques et prescripteurs français.

\section{Respect du lecteur}

Face à ce déferlement si peu habituel et violent du rouge et du noir sur le lecteur adolescent, seul un «respect du lecteur» (Delbrassine, 2006) peut garantir un sens moral en phase avec la doxa propre à la littérature jeunesse. L'autocensure est un moyen de mise en œuvre du respect, mais nous l'avons vu, Guéraud ne se contraint pas. Il met par contre en place un appareil littéraire qui instaure une distanciation du lecteur par rapport aux propos tenus. L'ironie se révèle être un moyen d'éloignement nécessaire et efficace. Guéraud en est parfaitement conscient puisqu'il appose en épigraphe à Déroute Sauvage ces quelques mots adressés au lecteur: «Je mentirais 
si je ne pensais pas déjà m'être amusé à raconter n'importe quoi pour faire marrer [les ados].» (2009, p.9) Il place ainsi l'ouvrage sous le signe de l'ironie et crée insensiblement une distance vis-à-vis de la violence gore et absurde du livre. Le lecteur n'a alors d'autre choix que de balancer entre effroi, dégoût et ironie, ce que confirme un critique: "Le lecteur (15 ans et plus), partagé entre panique et éclat de rire, se régale autant que lui. » (Abescat, 2007)

D'autres respects et procédés littéraires bien connus surgissent tout au long des textes, permettant une mise à distance du lecteur par rapport à la violence et aux fins relativement désespérées. Une combinaison de réalisme et de personnages surnaturels assoiffés de sang dans Déroute sauvage, la fantasy, le régime dictatorial aux armes imaginaires ou encore l'éloignement dans le temps futur dans La Brigade de l'œil constituent tous des éléments qui amènent le lecteur adolescent à s'interroger, par le biais du grotesque de la représentation, sur la violence proposée.

Cependant, ce respect du lecteur n'est pas toujours très clairement balisé dans le texte et se fait plus subtil, notamment si le genre du texte est réaliste. De ce point de vue, Je mourrai pas gibier constitue un livre à part, qui propose une autre violence, froide, mesurée, fortement dérangeante (les réactions et les interrogations de la critique ont été nombreuses ${ }^{7}$ ). Mortagne est certes un village au nom fictif mais il n'en reste pas moins un village qui pourrait exister. Le lecteur ne sort pas de la réalité, aucun phénomène surnaturel ou fantaisiste ne venant perturber la narration à la première personne. Les événements de l'actualité (Colombine, États-Unis, 2002 et

${ }^{7}$ Voir Lecture Jeune, « Des romans violents ? », no 128, décembre 2008, n. p. 
Utøya, Norvège, 2011) contribuent à renforcer l'aspect réaliste de la tuerie. Pourtant, là aussi, un respect du lecteur, sans doute plus subtil, est proposé par une structure narrative complexe avec l'usage de retours en arrière, s'adressant ainsi à un lecteur déjà expérimenté, capable de percevoir la différence entre réalité et imaginaire. Par cette mise à distance plus discrète du lecteur, l'auteur s'éloigne de la fonctionnalité didactique de la littérature jeunesse et s'écarte de l'horizon d'attente du prescripteur. Une crainte souvent évoquée dans les comptes rendus critiques des livres de Guéraud est celle du problème que pose cette violence évoquée sans pincettes. La violence guéraudienne ravive le débat $d u$ «peut-on tout dire à nos adolescents? » (voir Collectif. 2009) En traitant de la violence, et notamment de la violence gratuite, Guéraud prend le risque d'être censuré par différentes instances: par l'éditeur qui, soumis à la pression du public, s'en tient à une politique de prudence pour éviter au maximum un scandale aux conséquences souvent désastreuses économiquement, tout comme, éventuellement, par d'autres prescripteurs comme les diffuseurs, les bibliothécaires, les institutions scolaires, les parents, les médias, les libraires et la commission de censure chargée de faire appliquer la loi de 1949.

\section{La fin de l'innocence?}

La mise en scène d'un adolescent qui assassine sa propre famille à coup de fusil rompt avec une longue tradition de l'innocence de l'enfant, même si ce mythe se craquèle depuis déjà plusieurs décennies (pensons en particulier à la parution de Sa Majesté des Mouches [1954] de William Golding). Comme 
le soulignent Danielle Thaler et Alain Jean-Bart, les romans mettant en scène des enfants bourreaux perturbent

nos préjugés sur la psychologie juvénile et cass[ent] violemment l'image de l'enfance et de l'adolescence que nous voul[ons] admettre, ou derrière laquelle nous voul[ons] nous réfugier [...]. L'enfant victime demeurait un personnage clef des récits pour jeunes alors que l'enfant bourreau était refoulé aux portes de notre monde littéraire, un peu comme si notre littérature essayait de refouler les «monstres» que notre société était capable de fabriquer. » (2002, p. 148)

Fait de société, évolution du champ, l'enfant bourreau est un "personnage assez rare avant le dernier quart du $\mathrm{XX}^{\mathrm{e}}$ siècle » (Delbrassine, 2006, p. 319). Avec Je mourrai pas gibier, Guéraud se place dans la lignée de Golding ou de Cormier (The Chocolate War) en mettant en scène un adolescent qui se transforme en bourreau et renforce le caractère tabou de la violence. En s'attaquant de front au mythe de l'innocence, Guéraud ne fait qu'accentuer l'éventuelle résistance des prescripteurs face à un sujet aussi sensible et qui s'inquiètent des possibles retombées traumatisantes de la violence littéraire sur le lecteur adolescent.

\section{Vers un nouveau statut du lecteur?}

Ces trois romans de Guéraud dépeignent différentes nuances de rouge et de noir. Avec son style cru et la violence de ses propos, Guéraud devient un cas particulier de la littérature jeunesse en France. Peu d'auteurs français se sont risqués à écrire sur un sujet sensible de façon aussi descriptive et réaliste. Quelquesuns ont essayé de ne pas se restreindre, mais la critique s'est faite à son tour virulente. Des auteurs comme Thierry Lenain 
(La Fille du canal, Syros), Antoine Dôle Ue reviens de mourir, Sarbacane) ou Catherine Zambon (Kaïna-Marseille, Actes sud junior) ont été critiqués parce qu'ils dérangent par le choix de leurs sujets sensibles, joints à une écriture réaliste. Leurs éditeurs se sont fait pour certains «conseiller» par la commission de censure. La sensibilité du motif guéraudien pose problème en étant en décalage par rapport à la doxa française. Malgré le respect du lecteur atteint par des procédés littéraires connus, les prescripteurs s'interrogent sur la portée d'une telle violence sur le lecteur adolescent. En combinaison avec cette violence, la destruction du mythe de l'innocence de l'enfant enjoint certains prescripteurs à rendre plus difficile la diffusion de ces textes ; par exemple, un bibliothécaire ou un libraire aura peut-être plus de réticence à commander le livre ou à le mettre en valeur dans les rayonnages pour son public.

Enfin, l'une des conséquences indirectes des livres de Guéraud passe par l'évolution du statut du jeune lecteur. En effet, en refusant toute autocensure, Guéraud évacue l'aspect didactique traditionnellement attaché à la littérature jeunesse et envisage l'adolescent comme un lecteur indépendant et libre de ses choix, à l'image du lecteur adulte. La confiance dans le lecteur est finalement entière et les études d'habitude de lecture des jeunes ont bien montré que si l'adolescent n'est pas friand d'un livre, il arrêtera de lui-même ou n'empruntera pas de livres du même auteur à la bibliothèque. Celui qui achète et lit du Guéraud sait ce qu'il va y trouver : des cris, du sang et des pleurs. Guéraud a d'ailleurs trouvé son public malgré les réticences de certains prescripteurs. Cet auteur jeunesse n'est finalement pas méchant, il ne tue pas l'espoir, mais il considère l'adolescent comme un être capable de choisir, de critiquer et de réfléchir sur sa propre violence et sur celle de la société dans 
laquelle il vit. On peut y voir le plus grand respect porté à son lecteur. Pensant l'avoir oublié en écrivant, c'est bien lui que Guéraud met au premier plan.

\section{Bibliographie}

ABESCAT, Michel. (2007), «La Brigade de l'œil», Télérama, 26 novembre, <http://www.telerama.fr/livres/22445la brigade de 1 oeil.php $>$.

BAKHTINE, Mikhaïl. (1975 [1978]), Esthétique et théorie du roman, Paris, Gallimard, coll. « Tel ».

ColleCTIF. (2009), Peut-on tout dire (et tout montrer) dans les livres pour enfants?, Cahiers du CRILJ, $\mathrm{n}$ - 1 , novembre.

CRÉPIN, Thierry et Anne CRÉTOIS. (2003), « La presse et la loi de 1949, entre censure et autocensure ", Le Temps des médias, no 1, p. 56-57.

DELBRASSINE, Daniel. (2006), Les Romans pour adolescents aujourd'hui : écriture, thématiques et réception, SCÉRÉNCRDP et La Joie par les Livres - Centre national du livre pour enfants.

DESROCHES, Gisèle. (1994), «La violence dans la littérature québécoise pour la jeunesse », Lurelu, vol. 16, no 3, p. 39-41, http://www.erudit.org/culture/lurelu1081565/lurelu1108 967/12443ac.html?vue=resume $>$.

MARZANO, Michela (dir.). (2011), Dictionnaire de la violence, Paris, PUF, coll. « Quadrige ».

FERRIER, Bertrand. (2013), Les Méchants, des personnages comme il en faut, Cahiers du CRILJ, no 5, décembre.

FJELDBERG, Guri. (2011), « Le roman contemporain norvégien pour enfants et adolescents », Des prinçusses et des grands frères, Livres au trésor - Ambassade de Norvège. 
GIRARD, Quentin. (2013), «La censure, même pas morte! », Libération, 5 mai, $<$ http://next.liberation.fr/culture/2013/05/20/la-censurememe-pas-morte 904278>.

GIRARD, René. (1972), La Violence et le sacré, Paris, Grasset.

GuÉRAUD, Guillaume. (2006), Je mourrai pas gibier, Paris, Éditions du Rouergue, coll. « Doado noir ».

—. (2007), La Brigade de l'œil, Paris, Éditions du Rouergue, coll. « Doado noir».

—. (2009), Déroute sauvage, Paris, Éditions du Rouergue, coll. « Doado noir ».

JABKHIRO, Juliette. (2014), «La littérature jeunesse, sous surveillance depuis un siècle », Libération, 10 février, <http://next.liberation.fr/sexe/2014/02/10/la-litteraturejeunesse-sous-surveillance-depuis-un-siecle 979130>.

JauSS, Hans Robert. (1978), Pour une esthétique de la réception, Paris, Gallimard, coll. « Bibliothèque des Idées ».

Kristeva, Julia. (1980), Pouvoirs de l'horreur. Essai sur l'abjection, Paris, Seuil.

Rolland, Annie. (2008), Qui a peur de la littérature ado?, Paris, Éditions Thierry Magnier.

Rossignol, Véronique. (2006), sans titre, LivresHebdo, nº 629, 20 janvier, rubrique « Avant critiques ».

Éditions du Rouergue, Guillaume Guéraud, $<$ http://archive.wikiwix.com/cache/?url=http $\% 3 \mathrm{~A} \% 2 \mathrm{~F} \% 2 \mathrm{~F}$

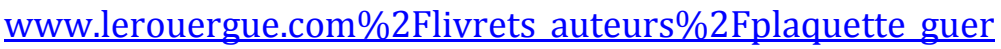
aud.pdf $>$.

THALER, D. et A. JEAN-BART. (2002), Les Enjeux du roman pour adolescents, Roman historique, roman miroir, roman d'aventures, Paris, L'Harmattan. 


\title{
Résumé
}

Dans le contexte de la littérature jeunesse, le traitement de la violence et de sa noirceur peut remettre en jeu les normes et l'horizon d'attente du lecteur adulte. Guillaume Guéraud est l'un des rares auteurs français écrivant des livres pour adolescents à refuser ces contraintes. À travers l'exemple de trois romans, cet article montre comment la violence prend place et se finalise parfois en une "fin non-heureuse ». Au-delà d'un défi aux normes traditionnelles en littérature pour adolescents, l'omniprésence du rouge et du noir dans les romans de Guéraud institue un statut plus élevé du lecteur adolescent et s'éloigne de la fonctionnalité didactique traditionnelle de la littérature de jeunesse.

\begin{abstract}
In the context of Children's Literature, the treatment of violence and its cruelty may challenge the norms as well as the adult reader's "horizon of expectation". Guillaume Guéraud is one of the few French authors for young adult's readers who chooses to ignore such norms. This article shows how violence is portrayed in three of his novels and how it can culminate in a "non-happy ending". The omnipresence of the red and black motifs in Guéraud's novels grants the teen reader a higher status, and transcends the didactic function often ascribed to adolescent literature.
\end{abstract}

